\title{
An easy and accurate respiratory rate monitor is necessary
}

\author{
Nicolas Marjanovic ${ }^{1}$ Olivier Mimoz ${ }^{1,2} \cdot$ Jérémy Guenezan ${ }^{1}$
}

Received: 10 July 2019 / Accepted: 16 July 2019 / Published online: 24 July 2019

(c) Springer Nature B.V. 2019

Patients admitted to acute care departments should be monitored very closely to improve their safety. Among the five common vital signs - pulse rate, blood pressure, pulse oximetry, respiratory rate $[R R]$ and temperature- $R R$ is probably the most in need of continuous monitoring. Indeed, as the National Institute for Health and Care Excellence has pointed out [1], RR is the most sensitive parameter to detect any clinical deterioration. In addition, RR monitoring can help to assess the severity of deterioration, to predict occurrence of serious adverse events and to estimate response to treatment [1].

$\mathrm{RR}$ is regulated by the central nervous system based on inputs from central and peripheral chemo-receptors. It varies so as to keep oxygen and carbon dioxide concentrations in the blood at levels close to normal range. Any disease responsible for hypoxemia, hypocapnia or hypercapnia, such as pneumonia, pulmonary embolism, acute heart failure or acute exacerbation of COPD, will be detected quickly by a modification of RR. During acute respiratory failure, RR is the best marker of disease severity and prognosis. It is more relevant than pulse oximetry, and when monitored, it can discriminate between different pathological conditions in respiratory disease [2], predict complications and exacerbations, assess response to treatment and anticipate occurrence of serious adverse events [3, 4].

In addition to conditions causing hypoxaemia, hypocapnia or hypercapnia, RR may also vary in diseases leading to metabolic acidosis [5] such as shock or renal failure. More generally, RR is the vital sign with the highest performance to identify patients requiring intensive care admission [6] or those visiting an emergency department and requiring hospital admission [7]. Variations in RR are much greater

Olivier Mimoz

olivier.mimoz@wanadoo.fr

1 Emergency Department and Prehospital Care, University Hospital of Poitiers, Rue de la Milétrie, 86000 Poitiers, France

2 School of Medicine and Pharmacy, University of Poitiers, Rue de la Milétrie, 86000 Poitiers, France than changes in heart rate or systolic blood pressure and consequently more susceptible to distinguish stable patients from patients at risk [8]. In addition, RR changes are faster and easier to measure than prognostic scores to be calculated or biological tests to be performed. Lastly, RR monitoring is useful in the diagnosis of specific iatrogenic adverse events, of which the most frequent is respiratory depression during the postoperative period or after drug poisoning when drugs or substances that depress ventilation are used.

Despite its undeniable utility and reliability, RR is rarely recorded because of the difficulty in having it measured and continuously monitored [9]. Several methods are available and all have advantages and drawbacks. Manual counting by patient observation for 30 or $60 \mathrm{~s}$ is the most common. It is very simple to implement but time-consuming and cannot provide continuous monitoring. While reliable, it is difficult to carry out in overcrowded units with risk of measurement error $[10,11]$. Impedance pneumography is a commonly used method for continuous and automated measurement of $\mathrm{RR}$. It is based on the use of alternating current between two surface electrodes that record chest movements. However, any movement such as tremors or closed glottis breathing efforts (as in obstructive apnea) causes impedance variation and thereby detects false breathing cycles [13, 14]. Capnometry is considered the gold standard in patients under mechanical ventilation but is difficult to perform in nonintubated patients [8].

Alternative methods have recently been developed to overcome these limitations. They include acoustic transducers, humidity sensors and infrared thermal cameras [12]. Acoustic transducers measure RR with high accuracy but cough, sneeze, snore or cries may interfere with performance [13]. Infrared thermal cameras are relatively new systems and seem reliable, but they are susceptible to body motions and different interactions between patient and system [14].

In this issue, Hurtato and his colleagues reported the value of a new device using a continuous and non-invasive temperature-based respiratory monitoring system. It consists of buccal and nasal thermal collectors installed face to oral and nasal airflow. Temperature variations of the respiratory 
flow are collected through thermal sensor resistance and converted into continuous electrical signal to obtain relevant respiratory flow parameter. RR is estimated through an average crossing algorithm adding nasal and oral signals, allowing for accurate estimation.

In a group of 20 healthy volunteers, the authors simultaneously compared the RR value obtained with their device or visual counting. Results were promising. Average RR difference between the monitor and visual counting was 0.4 breaths per minute (BPM) (95\% CI -0.5 to $1.3 \mathrm{BPM})$ and the system seems able to detect low RR. However, limitations should be acknowledged. First, even though overall performance of device does not seem to be impacted, the authors highlighted the poor performance of the oral sensor. Second, patient tolerance and comfort should be explored in larger studies to determine the usability of the device in a clinical setting. Finally, the performance of this device in several pathological conditions needs to be explored in further studies.

Continuous RR monitoring is essential as a means of improving the safety of patients admitted to acute care units. Current methods all present drawbacks that limit their value. The development of new technologies is necessary. Researchers and manufacturers should work together to develop reliable technology regardless of the patient's clinical condition.

\section{Compliance with ethical standards}

Conflict of interest Authors have no competing interest to declare.

\section{References}

1. National Institute for Health and Care Excellence. Acutely ill adults in hospital: recognising and responding to deterioration. 2007.

2. Lapi S, Lavorini F, Borgioli G, et al. Respiratory rate assessments using a dual-accelerometer device. Respir Physiol Neurobiol. 2014;1191:60-6.
3. Fieselmann JF, Hendryx MS, Helms CM, et al. Respiratory rate predicts cardiopulmonary arrest for internal medicine inpatients. J Gen Intern Med. 1993;8:354-60.

4. Churpek MM, Yuen TC, Park SY, et al. Derivation of a cardiac arrest prediction model using ward vital signs*. Crit Care Med. 2012;40:2102-8.

5. West J, editor. Respiratory physiology: the essentials. 9th ed. Philadelphia: Wolters Kluwer/Lippincott Williams \& Wilkins; 1990.

6. Cuthbertson BH, Boroujerdi M, McKie L, et al. Can physiological variables and early warning scoring systems allow early recognition of the deteriorating surgical patient? Crit Care Med. 2007;35:402-9.

7. Lam T, Mak P, Siu W, et al. Validation of a modified early warning score (mews) in emergency department observation ward patients. Hong Kong J Emerg Med. 2006;13:24-30.

8. Subbe CP, Kinsella S. Continuous monitoring of respiratory rate in emergency admissions: evaluation of the RespiraSense ${ }^{\mathrm{TM}}$ sensor in acute care compared to the industry standard and gold standard. Sensors (Basel). 2018;18:2700.

9. Cretikos MA, Bellomo R, Hillman K, et al. Respiratory rate: the neglected vital sign. Med J Aust. 2008;188:657-9.

10. Kellett J, Li M, Rasool S, et al. Comparison of the heart and breathing rate of acutely ill medical patients recorded by nursing staff with those measured over $5 \mathrm{~min}$ by a piezoelectric belt and ECG monitor at the time of admission to hospital. Resuscitation. 2011;82:1381-6.

11. Lovett PB, Buchwald JM, Stürmann K, et al. The vexatious vital: neither clinical measurements by nurses nor an electronic monitor provides accurate measurements of respiratory rate in triage. Ann Emerg Med. 2005;45:68-76.

12. Liu H, Allen J, Zheng D, et al. Recent development of respiratory rate measurement technologies. Physiol Meas. 2019. https://doi. org/10.1088/1361-6579/ab299e.

13. Guechi Y, Pichot A, Frasca D, et al. Assessment of noninvasive acoustic respiration rate monitoring in patients admitted to an Emergency Department for drug or alcoholic poisoning. J Clin Monit Comput. 2015;29:721-6.

14. Hochhausen N, Barbosa Pereira C, Leonhardt S, et al. Estimating respiratory rate in post-anesthesia care unit patients using infrared thermography: an observational study. Sensors (Basel). 2018;18:1618.

Publisher's Note Springer Nature remains neutral with regard to jurisdictional claims in published maps and institutional affiliations. 